\title{
Androgen Receptor as a Biomarker of Oral Squamous Cell Carcinoma Progression Risk
}

\author{
CEDNA TOMASOVIC-LONCARIC ${ }^{1 *}$, ALEKSANDRA FUCIC $^{2 *}$, ANA ANDABAK ${ }^{3}$, MATEJ ANDABAK $^{1}$, \\ MARCELLO CEPPI ${ }^{4}$, MARCO BRUZZONE $^{4}$, DANKO VRDOLJAK ${ }^{5}$ and VANJA VUCICEVIC-BORAS ${ }^{3}$ \\ ${ }^{1}$ Clinical Hospital "Dubrava", Zagreb, Croatia; \\ ${ }^{2}$ Institute for Medical Research and Occupational Health, Zagreb, Croatia; \\ ${ }^{3}$ School of Dentistry, University of Zagreb, Zagreb, Croatia; \\ ${ }^{4}$ Ospedale Policlinico San Martino, Genova, Italy; \\ ${ }^{5}$ Clinic for tumours, Clinical Hospital Center Sisters of Mercy, Zagreb, Croatia
}

\begin{abstract}
Background/Aim: Oral squamous cell carcinoma (OSCC) is a cancer with poor prognosis due to therapy resistance, locoregional recurrences, and distant metastases. There is on increased interest in profiling the androgen receptor $(A R)$ in cancer biology. The aim of this study was to compare AR and Ki-67 levels in the neoplastic epithelium and stroma between non-metastatic and metastatic stages of OSCC. Patients and Methods: Tissue specimens of 101 nonmetastatic and 95 metastatic OSCC patients were analyzed by immunohistochemistry. Results: More than 20\% of ARpositive cytoplasmic staining of OSCC epithelium was significantly associated with nuclear AR levels in the epithelium and increased $A R$ levels in the stroma. In metastatic OSCC patients, Ki-67 was significantly higher than in non-metastatic OSCC patients. Conclusion: More than 20\% of AR-positive cytoplasmic staining in neoplastic OSSC epithelium is a significant predictor of OSCC progression risk.
\end{abstract}

Oral squamous cell carcinoma (OSCC) is the most common type of oral cavity cancer that has poor prognosis due to therapy-resistant, locoregional recurrences and distant metastases. Despite significant advancements in therapy, it is still a major concern worldwide. The five-year survival rate of oral cancer is $62.1 \%$ (1). Therapy of OSCC includes

\footnotetext{
*These Authors contributed equally to the preparation of the manuscript.
}

Correspondence to: Aleksandra Fucic, Institute for Medical Research and Occupational Health, Zagreb, Ksaverska c 2, Croatia. Tel: +385 14682500,e-mail: afucic@imi.hr

Key Words: Androgen receptor, Ki67, oral squamous cell carcinoma, metastasis, biomarker. surgery and radiotherapy. The identification of the high-risk subset of OSCC is critical for producing a more successful therapy $(2,3)$.

The significance of androgen receptors (AR) in the aetiology and progression of cancer has been recognized and is considered a novel potential target for better diagnostics and novel therapy options. AR levels are known to be associated with progression in several cancer types (4-8). However, there is a clear gap in data on the association of AR levels with OSCC. In vitro experiments and a study with patients have shown that OSCC cells express AR and that AR is critical for promoting cell growth $(9,10)$.

$\mathrm{AR}$ is a ligand-activated transcription factor, which in the absence of a hormone ligand (testosterone and dihydrotestosterone), is located preferentially in the cytoplasm. After activation, cytoplasmic AR translocates into the nucleus, where the AR-ligand complex binds androgen response elements in the gene promoter and enhancer regions of multiple target genes (11). In breast cancer, it has been shown that higher expression of $\mathrm{AR}$ in the nucleus than in the cytoplasm is associated with worse prognosis due to the AR activation of tumour genes such as vimentin (12).

Tumour stroma as a significant component of neoplastic progression has also been associated with AR levels (13, 14). An activated tumour stroma contains activated fibroblasts that induce the production of growth factors, cytokines, chemokines and metalloproteinases, which have roles in cancer cell differentiation, proliferation and local invasion (15).

The aim of this study was to investigate (a) the association between AR levels in the cytoplasm and nucleus of the OSCC neoplastic epithelium of non-metastatic and metastatic OSCC, (b) the levels of AR in stroma, (c) the association between epithelium AR and stroma AR and (d) the relationship of AR levels in non-metastatic and metastatic OSCC with Ki-67 activity. Results of this study should lead 
to the identification of new tools to recognize a subgroup of patients with a higher risk of metastasis.

\section{Patients and Methods}

In this study, 101 non-metastatic and 95 metastatic OSCC patients (78.1\% males and $29.9 \%$ females) were analysed. Stages of OSSC cancer ranged from T1N0 to T4N2. The mean age of patients was $62.59 \pm 11.24$ years (range $=31-92$ years). All of the performed procedures were in accordance with the ethical standards of the 1964 Declaration of Helsinki I. The study was approved by the Ethics Committee of Clinical Hospital "Dubrava", Zagreb, Croatia. Tissue specimens used in the current study were part of the hospital's tissue archive.

Resected tissue specimens were formalin-fixed, paraffinembedded, and cut on a microtome to form tissue sections (thickness $5 \mu \mathrm{m}$ ). Immunohistochemical analyses were performed using a streptavidin-biotin method on a DAKO autostainer with monoclonal antibodies for AR (AR 441, DAKO, 1:50 dilution) and Ki-67 (K 8000, DAKO, 1:75 dilution) as a proliferative marker. Monoclonal antibodies were used after heat induced epitope retrieval (PT-LINK, $97 \mathrm{C}$ ). For visualisation Polymer Conjugate Envision FLEX with DAB chromogen (K 8000, DAKO, Glostrup, Denmark) was applied. The immunohistochemistry was performed on Dako Autostainer plus. Sections were counterstained with hematoxylin and eosin. As a positive control prostate tissue was used. Immunoreactivity reactions were determined in the cytoplasm and nucleus of neoplastic epithelium and stromal components under the magnification of $400 \mathrm{X}$ for a total of 1,000 tumour cells.

Statistics. A logistic regression model was applied to the binary outcome metastatic stage no/yes to estimate the predictability of the AR level in cytoplasm $\geq 20 \%$ with respect to $<20 \%$ level on the risk of the occurrence of metastases, adjusting by age and gender. To investigate the relationship between Ki67 and cytoplasmic AR in occurrence of metastasis, the log-normal regression model was applied. This statistical model was also used to test the association between nuclear AR levels in epithelial and stromal cells with cytoplasmic AR.

\section{Results}

Table I shows the profiles of the studied patients. More than $20 \%$ of cytoplasmic epithelium AR positivity was significantly associated with an increase in AR nuclear positivity of neoplastic epithelium cells and stromal cells. Thus, patients with cytoplasmic $A R \geq 20 \%$ in epithelial cells had an average nuclear AR levels higher 2.57 times (95\%CI=1.92-3.43; $p<0.001)$ than patients with cytoplasmic $\mathrm{AR}<20 \%$ adjusting for age, gender and occurrence of metastasis; likewise cytoplasmic AR more than $20 \%$ is significantly associated with increased AR levels in stromal cells $(p<0.001)$.

Results from the logistic regression model applied to the binary outcome metastatic stage no/yes show that males have more risk of metastasis than women $(\mathrm{OR}=2.11$; $95 \% \mathrm{CI}=1.01-4.39 ; p=0.047)$ and a frequency of $\mathrm{AR}$ receptors in the cytoplasm greater than $20 \%$, is associated
Table I. Description of the characteristics of patients examined.

\begin{tabular}{|c|c|c|}
\hline & \multicolumn{2}{|c|}{$\mathrm{N}(\%)$} \\
\hline \multicolumn{3}{|l|}{ Gender } \\
\hline 1 & \multicolumn{2}{|c|}{43 (21.9) } \\
\hline 2 & \multicolumn{2}{|c|}{$153(78.1)$} \\
\hline \multicolumn{3}{|l|}{ Metastatic } \\
\hline No & \multicolumn{2}{|c|}{$101(51.5)$} \\
\hline Yes & \multicolumn{2}{|c|}{$95(48.5)$} \\
\hline & Mean (SD) & Min-Max \\
\hline $\mathrm{AGE}$ & $62.59(11.24)$ & $31-92$ \\
\hline ER_EPITEL & $0.89(5.46)$ & $0-70$ \\
\hline ER_STROMA & $0.09(0.81)$ & $0-10$ \\
\hline AR_EPITEL & $4.48(8.83)$ & $0-80$ \\
\hline AR_STROMA & $3.04(7.07)$ & $0-42$ \\
\hline KI67_EPITEL & $47.54(15.10)$ & $15-90$ \\
\hline KI67_STROMA & $13.01(7.86)$ & $2-36$ \\
\hline
\end{tabular}

with the occurrence of metastasis $(\mathrm{OR}=1.87 ; 95 \% \mathrm{CI}=1.03$ $3.40 ; p=0.040$ ) (Table II).

In metastatic OSCC patients with more than $20 \%$ of cytoplasmic AR, a significant increase in $\mathrm{Ki}-67$ by $28 \%$ was observed in the epithelium compared to the non-metastatic OSCC group (Table III).

\section{Discussion}

The results of this study showed for the first time that more than 20\% AR-positive cytoplasmic staining in the neoplastic epithelium can be used as a predictor of a significantly increased risk of metastasis in OSCC patients. This cut-off value is significantly associated with an increase in the AR nuclear positivity of neoplastic epithelium cells and increased levels of AR in stromal cells. Additionally, in metastatic OSCC patients with more than $20 \%$ cytoplasmic AR positive cells, there was a significant (28\%) increase of $\mathrm{Ki}-67$ in the epithelium compared to non-metastatic OSCC patients. Although the number of cases was small, our results showed that males have a higher risk of metastasis than women.

During the last decade, a significant increase in the survival rate has been reported for patients with OSCC which is an aggressive cancer type with limited therapeutic options and late diagnostics, (16). Therefore, it is of great importance to introduce biomarkers that will enable therapy planning and recognition of subgroups that are at higher risk of metastasis and may need specific follow up after therapy.

Current data showed that oestrogen and testosterone play a significant role in the aetiology and progression of all cancer types $(7,17,18)$. Potency of hormonal therapy in different cancer types other than breast, testis, ovarian and 
Table II. Results of the logistic regression model applied to the binary outcome metastatic stage no/yes.

\begin{tabular}{lccc}
\hline Metastatic stage no/yes & Odds ratio & $95 \% \mathrm{CI}$ & $p$-Value \\
\hline Age & 1.00 & $0.97-1.03$ & 0.875 \\
Females & 1.00 & - & - \\
Males & 2.11 & $1.01-4.39$ & 0.047 \\
Cytoplasmic AR<20 & 1.00 & - & - \\
Cytoplasmic AR $\geq 20$ & 1.87 & $1.03-3.40$ & 0.040 \\
\hline
\end{tabular}

prostate cancer was already recognized several decades ago. However, only recently the mechanisms of hormonal therapy were elucidated, providing the foundations for more efficient hormonal therapy, especially in the context of immunotherapy and personalized medicine (19-21).

The AR is a transcription factor with broad tissue distribution and a major mediator of cellular functions and homeostasis. AR resides in an inactive state in the cytoplasm in complexes with molecular chaperones such as HSP90. Activation of AR by testosterone results in its translocation from the cytoplasm to the nucleus and ultimately regulation of the cellular transcriptional profile (22). Our findings in this study are in concordance with these mechanisms as presence of increased AR levels in cytoplasm of neoplastic epithelium are associated with increased nuclear positivity and indicators of cancer progression such as increased AR positivity in stroma cells and increased levels of Ki67 in metastatic patients.

One of possible mechanisms which bridge AR and Ki67 activities could involve kallikrein as has been shown in head and neck squamous cell carcinoma cells (23). Glandular kallikrein 1(KLK1) which is specific for head and neck cancer is a target of AR (24). KLK1 released from tumour cells enzymatically generates mitogenic kinins (25) and facilitates the dissemination of tumour cells (26). Mechanisms similar to those involved in kalilikrein 7mediated increase in ki67 levels in colon cancer (27) may also be present in OSCC.

The stroma is composed of fibroblasts, smooth muscle cells, immune cells, lymphatics, and an extracellular matrix rich in signal molecules such as cytokines, growth factors, hormones, enzymes, etc. In addition to cell-cell interaction, these molecules mediate communication, through multidirectional signalling, between stromal and epithelial compartments. During carcinogenesis, significant changes are present in stroma including loss of smooth muscle cells and activation of myofibroblasts, termed cancer associated fibroblasts (CAFs). Activated cancer stroma enables and facilitates migration of neoplastic cells and disease progression. Targeting the stroma
Table III. Estimates from the log-normal regression model applied to Ki67 in the epithelium to verify the relationship with cytoplasmic AR and occurrence of metastasis.

\begin{tabular}{lcc}
\hline & \multicolumn{2}{c}{ Cytoplasmic AR } \\
\cline { 2 - 3 } & $\geq 20 \%$ & $<20 \%$ \\
\hline Metastatic stage & Mean ratio $(95 \% \mathrm{CI})$ & Mean ratio $(95 \% \mathrm{CI})$ \\
No & 1.00 & $1.10(0.96-1.26)$ \\
Yes & $1.28(1.10-1.49)$ & $1.13(0.97-1.30)$ \\
\hline
\end{tabular}

as a key neoplastic micromilleu has been suggested for decades but only recently have its role and mechanisms been better understood, thus opening options for complementary therapy development $(13,28,29)$.

Current knowledge on the dynamics of AR and its interplay with stroma is almost exclusively based on data collected for prostate cancer and anecdotal studies on breast cancer (30-33). However, stromal-epithelial interactions have been shown to orchestrate AR signalling and tumorigenesis (34). Thus, it has been suggested that AR expressed in the stroma of oesophageal carcinoma may induce paracrine effects following stimulation by androgens possibly via fibroblast growth factors (35). The stroma has been already suggested as a prognostic tool in OSCC (36). Thus, in addition to confirming $\mathrm{AR}$ as a prognostic biomarker for metastasis, our results highlight the need of inclusion of a cut off value of $20 \%$ AR positivity in epithelial cytoplasm in patho-histological diagnostics.

Our finding that male OSCC patients have higher risk of metastasis than women is in concordance with sex-specific incidence and survival rate in different cancer types. Such difference may originate from differential susceptibility to environmental factors but also from differential effects of therapy. $(37,38)$. This is very important in preventive measures and therapy planning.

In conclusion, our results gave, for the first time, a cut off value of $20 \%$ AR positivity in the cytoplasm of neoplastic OSCC epithelium as a prognostic biomarker for metastasis risk. Additionally, it showed a clear association between AR levels in epithelium and stroma, and highlighted the impact of this interaction on neoplastic proliferation potency in OSCC. These findings should be further investigated for future therapeutic treatments. Introduction of $\mathrm{AR}$ as a biomarker of OSCC progression in pathohistological diagnostics is suggested.

\section{Conflicts of Interest}

The Authors declare no conflict of interests regarding this study. 


\section{Authors' Contributions}

CTL and AF perform analysis and drafted the manuscript. AM, DV and $\mathrm{MM}$ selected patients, collected clinical data and drafted the manuscript. MC and MB performed statisctical analysis, data interpretation and drafted the manuscript. VVB provided design of the study and drafted the manuscript.

\section{Acknowledgements}

This study was funded by the Croatian Science Foundation (project "The role of androgen and oestrogen receptors in the stromal activation of patients with head and neck cancers and its influence on patients surviva" (No:IP-2014-09-6985).

\section{References}

1 Ferlay J, Soerjomataram I, Ervik M, Dikshit R, Eser S, Mathers C, Rebelo M, Parkin DM, Forman D and Bray F: Globocan 2012 v1.0, cancer incidence and mortality worldwide. IARC CancerBase, 2016.

2 Katsoulakis E, Leeman JE, Lok BH, Shi W, Zhang Z, Tsai JC, McBride SM, Sherman EJ, Cohen M, Wong R, Ganly I, Lee NY and Riaz N: Long-term outcomes in oral cavity squamous cell carcinoma with adjuvant and salvage radiotherapy after surgery. The Laryngoscope 128(11): 2539-2545, 2018. PMID: 29637571. DOI: $10.1002 /$ lary.27191

3 Liu T, Chua B and Batstone M: Postoperative radiotherapy for oral squamous cell carcinoma with histologic risk factors: are we over-treating? J Oral Maxillofacial Surg 76(7): 1565-1570, 2018. PMID: 29438656. DOI: 10.1016/j.joms.2018.01.014

4 Mandel A, Larsson P, Sarwar M, Semenas J, Syed Khaja AS and Persson JL: The interplay between AR, EGF receptor and MMP9 signaling pathways in invasive prostate cancer. Mol Med 24(1): 34, 2018. PMID: 30134822. DOI: 10.1186/s10020-0180035-4

5 Kraby MR, Valla M Opdahl S, Haugen OA, Sawicka JE, Engstrom MJ and Bofin AM: The prognostic value of androgen receptors in breast cancer subtypes. Breast Cancer Res Treat 172(2): 283-296, 2018. PMID: 30109519. DOI: 10.1007/s10549018-4904-x

6 Zhang BG, Du T, Zang MD, Chang Q, Fan ZY, Li JF, Yu BQ, Su LP, Li C, Yan C, Gu QL, Zhu ZG, Yan M and Liu B: Androgen receptor promotes gastric cancer cell migration and invasion via AKT-phosphorylation dependent upregulation of matrix metalloproteinase 9. Oncotarget 5(21): 1, 2014. PMID: 25301736. DOI: 10.18632 /oncotarget 2513

7 Jukic Z, Radulovic P, Stojković R, Mijic A, Grah J, Kruslin B, Ferencic $Z$ and Fucic A: Gender difference in distribution of estrogen and androgen receptors in intestinal-type gastric cancer. Anticancer Res 37(1): 197-202, 2017.PMID: 28011491. DOI: 10.21873/anticanres.11306

8 Huang Q, Sun Y, Ma X, Gao Y, Li X, Niu Y, Zhang X and Chang C: Androgen receptor increases hematogenous metastasis yet decreases lymphatic metastasis of renal cell carcinoma. Nat Commun 8(1): 918, 2017. PMID: 29030639. DOI: 10.1038/ s41467-017-00701-6

9 Wu TF, Luo FJ, Chang YL, Huang CM, Chiu WJ, Weng CF, Hsu YK and Yuan TC: The oncogenic role of androgen receptors in promoting the growth of oral squamous cell carcinoma cells. Oral Dis 1(3): 320-327, 2012. PMID: 25040852. DOI: 10.1111/ odi. 12272

10 Marocchio LS, Giudic F, Corrêa L, Pinto Junior Ddos S and de Sousa SO: Oestrogens and androgen receptors in oral squamous cell carcinoma. Acta Odontol Scand 71(6): 1513-1519, 2013. PMID: 24066884. DOI: 10.3109/00016357.2013.775335

11 Jenster G, Trapman J and Brinkmann A: Nuclear import of the human androgen receptor. Biochem J 293: 761-768, 1993. PMID: 8352744. DOI: 10.1042/bj2930761

12 Feng J, Li L, Zhang N, Liu J, Zhang L, Gao H, Wang G, Li Y, Zhang Y, Li X, Liu D, Lu J and Huang B: Androgen and AR contribute to breast cancer development and metastasis: an insight of mechanisms. Oncogene 36(20): 2775-2790, 2017. PMID: 27893717. DOI: 10.1038/onc.2016.432

13 Chan JSK, Sng MK, Teo ZQ, Chong HC, Twang JS and Tan NS: Targeting nuclear receptors in cancer-associated fibroblasts as concurrent therapy to inhibit development of chemoresistant tumors. Oncogene 37(2): 160-173, 2018. PMID: 28892046. DOI: $10.1038 /$ onc.2017.319

14 Kikuchi K, McNamara KM, Miki Y, Moon JY, Choi MH, Omata F, Sakurai M, Onodera Y, Rai Y, Ohi Y, Sagara Y, Miyashita M, Ishida T, Ohuchi $\mathrm{N}$ and Sasano $\mathrm{H}$ : Effects of cytokines derived from cancer-associated fibroblasts on androgen synthetic enzymes in estrogen receptor-negative breast carcinoma. Breast Cancer Res Treat 166(3): 709-723, 2017. PMID: 28831645. DOI: $10.1007 / \mathrm{s} 10549-017-4464-5$

15 Karagiannis GS, Poutahidis T, Erdman SE, Kirsch R, Riddell RH and Diamandis E: Cancer-associated fibroblasts drive the progression of metastasis through both paracrine and mechanical pressure on cancer tissue. Mol Cancer Res 10(11): 1403-1418, 2012. PMID: 23024188. DOI: 10.1158/1541-7786.MCR-120307

16 Majumdar B, Patil S, Sarode SC, Sarode GS and Rao RS: Clinico-pathological prognosticators in oral squamous cell carcinoma. Translational Res Oral Oncol 2: 1-14, 2017. DOI: 10.1177/2057178X17738912

17 Allain EP, Venzl K, Caron P, Turcotte V, Simonyan D, Gruber M, Le T, Lévesque E, Guillemette C and Vanura K: Sexdependent association of circulating sex steroids and pituitary hormones with treatment-free survival in chronic lymphocytic leukemia patients. Ann Hematol 97(9): 1649-1661, 2018. PMID: 29781039. DOI: 10.1007/s00277-018-3356-Z

18 Fucic A, Gamulin M, Ferencic Z, Katic J, Krayer von Krauss M, Bartonova A and Merlo DF: Environmental exposure to xenoestrogens and oestrogen related cancers: reproductive system, breast, lung, kidney, pancreas, and brain. Environ Health 11: S8, 2012. PMID: 22759508. DOI: 10.1186/1476-069X-11-S1-S8

19 Konduri S, Schwarz MA, Cafasso D and Schwarz RE: Androgen receptor blockade in experimental combination therapy of pancreatic cancer. J Surg Res 142(2): 378-386, 2007. PMID: 17559882. DOI: $10.1016 /$ j.jss.2006.09.034

20 Greenway BA: Androgen receptor-blocking agents: potential role in pancreatic cancer. Drugs Aging 17(3): 161-163, 2000. PMID: 11043816. DOI: 10.2165/00002512-200017030-00001

21 Meikle AW, Cardoso de Sousa JC, Ward JH, Woodward M and Samlowski WE: Reduction of testosterone synthesis after high dose interleukin-2 therapy of metastatic cancer. J Clin Endocrinol Metab 73(5): 931-935, 1991. PMID: 1834690. DOI: 10.1210/jcem-73-5-931 
22 Leach DA and Buchanan G: Stromal androgen receptor in prostate cancer development and progression. Cancers (Basel) 9(1): 10, 2017. PMID: 28117763. DOI: 10.3390/cancers9010010

23 Beck C, Piontek G, Haug A, Bas M, Knopf A, Stark T, Mißlbeck M, Rudelius M, Reiter R, Brandstetter M and Pickhard A: The kallikrein-kinin-system in head and neck squamous cell carcinoma (HNSCC) and its role in tumour survival, invasion, migration and response to radiotherapy. Oral Oncol 48(12): 1208-1219, 2012. PMID: 22748451. DOI: 10.1016/ j.oraloncology.2012.06.001

24 Sun F, Chen H, Yang WLX, Wang X, Jiang R, Guo Z, Chen H, Huang J, Borowsky AD and Qiu Y: Androgen receptor splice variant AR3 promotes prostate cancer via modulating expression of autocrine/paracrine factors. J Biol Chem 289(3): 1529-1539, 2014. PMID: 24297183. DOI: 10.1074/jbc.M113.492140

25 Dlamini Z and Bhoola KD: Upregulation of tissue kallikrein, kinin $\mathrm{B} 1$ receptor, and kinin $\mathrm{B} 2$ receptor in mast and giant cells infiltrating oesophageal squamous cell carcinoma. J Clin Pathol 58(9): 915-922, 2005. PMID: 16126870. DOI: 10.1136/jcp.2004. 021444

26 Lawrence MG, Lai J and Clements JA: Kallikreins on steroids: structure, function, and hormonal regulation of prostate-specific antigen and the extended kallikrein locus. Endocrine Rev 31(4): 407-444, 2010. PMID: 20103546. DOI: 10.1210/er.2009-0034

27 Walker F, Nicole P, Jallane A, Soosaipillai A, Mosbach V, Oikonomopoulou K, Diamandis EP, Magdolen V and Darmoul D: Kallikrein-related peptidase 7 (KLK7) is a proliferative factor that is aberrantly expressed in human colon cancer. Biol Chem 395(9): 1075-1086, 2014. PMID: 25153388. DOI: 10.1515/hsz2014-0142

28 Santi A, Kugeratsk FG and Zanivan S: Cancer associated fibroblasts: The architects of stroma remodeling. Proteomics 18(5-6): e1700167, 2018. PMID: 29280568. DOI: 10.1002/pmic. 201700167

29 Munoz J, Jennifer J, Wheler JJ and Kurzrock R: Androgen receptors beyond prostate cancer: an old marker as a new target. Oncotarget 6(2): 592-603, 2015. PMID: 25595907. DOI: 10.18632 /oncotarget.2831

30 Harvell DM, Richer JK, Singh MN, Singh M, Spoelstra N, Finlayson C, Borges VF, Elias AD and Horwitz KB: Estrogen regulated gene expression in response to neoadjuvant endocrine therapy of breast cancers: tamoxifen agonist effects dominate in the presence of an aromatase inhibitor. Breast Cancer Res Treat 112: 489-501, 2008. PMID:18338247. DOI: 10.1007/s10549008-9923-6
31 Farmer P, Bonnefoi H, Becette VM, Tubiana-Hulin M, Fumoleau P, Larsimont D, Macgrogan G, Bergh J, Cameron D, Goldstein D, Duss S, Nicoulaz AL, Brisken C, Fiche M, Delorenzi M and Iggo R: Identification of molecular apocrine breast tumours by microarray analysis. Oncogene 24: 4660-4671, 2005. PMID: 15897907. DOI: $10.1038 /$ sj.onc. 1208561

32 Tang D, Xu S, Zhang Q and Zhao W: The expression and clinical significance of the androgen receptor and E-cadherin in triple-negative breast cancer. Med Oncol 29: 526-533, 2012. PMID: 21519872. DOI:10.1007/s12032-011-9948-2

33 Koo JS, Jung W and Jeong J: The predictive role of E-cadherin and androgen receptor on in vitro chemosensitivity in triplenegative breast cancer. Japanese J Clin Oncol 39: 560-568, 2009. PMID: 19531543. DOI: 10.1093/jjco/hyp065

34 Nieto CM, Rider LC and Cramer SD: Influence of stromalepithelial interactions on androgen action. Endocr Relat Cancer 21: T147-T160, 2014. PMID: 24872510. DOI: 10.1530/ERC-140138

35 Beck C, Piontek G, Haug A, Bas M, Knopf A, Stark T, Mißlbeck M, Rudelius M, Reiter R, Brandstetter M and Pickhard A: The kallikrein-kinin-system in head and neck squamous cell carcinoma (HNSCC) and its role in tumour survival, invasion, migration and response to radiotherapy. Oral Oncol 48(12): 1208-1219, 2012. PMID: 22748451. DOI: 10.1016/ j.oraloncology.2012.06.001

36 Ha SY, Yeo SY, Xuan YH and Kim SH: The prognostic significance of cancer-associated fibroblasts in esophageal squamous cell carcinoma. PLoS One 9(6): e99955, 2014. PMID: 24945657. DOI: 10.1371/journal.pone.0099955

37 Radkiewicz C, Johansson ALV, Dickman PW, Lambe M and Edgren G: Sex differences in cancer risk and survival: A Swedish cohort study. Eur J Cancer 84: 130-140, 2017. PMID: 28802709. DOI: 10.1016/j.ejca.2017.07.013

38 Dorak MT and Karpuzoglu E: Gender differences in cancer susceptibility: an inadequately addressed issue. Front Genet 3: 268, 2012. PMID: 23226157. DOI:10.3389/fgene.2012.00268

Received May 25, 2019

Revised June 14, 2019

Accepted June 18, 2019 